\title{
Reactive oxygen species-mediated activation of the Src-epidermal growth factor receptor-Akt signaling cascade prevents bortezomib-induced apoptosis in hepatocellular carcinoma cells
}

\author{
JINLIN HOU ${ }^{1}$, ANGUO CUI ${ }^{1}$, PEIYING SONG ${ }^{1}$, HUI HUA ${ }^{2}$, TING LUO $^{3}$ and YANGFU JIANG ${ }^{1}$ \\ ${ }^{1}$ State Key Labaoratory of Biotherapy, Section of Oncogene, Chengdu; ${ }^{2}$ Laboratory of Stem Cell Biology and \\ ${ }^{3}$ Cancer Center, West China Hospital, Sichuan University, \\ Chengdu, Sichuan 610041, P.R. China
}

Received December 20, 2013; Accepted July 25, 2014

DOI: $10.3892 / \mathrm{mmr} .2014 .2736$

\begin{abstract}
Proteasomes are essential for numerous cellular processes, including the cell cycle, regulation of gene expression and responses to cellular stress. Proteasome inhibitors are promising anticancer agents. The proteasome inhibitor bortezomib effectively suppresses certain types of cancer, including multiple myeloma and mantle cell lymphoma. However, bortezomib poorly inhibits solid tumors, including hepatocellular carcinoma. The activation of Akt represents an adaptive response that prevents bortezomib-induced cell apoptosis. In the present study, bortezomib induced phosphorylation of EGFR, Src and Akt in hepatoma cells and inhibition of Src reduced bortezomib-induced EGFR and Akt phosphorylation. Treatment of hepatoma cells with bortezomib led to an increase in the levels of intracellular reactive oxygen species (ROS). The ROS scavenger N-acetyl-L-cysteine inhibits bortezomib-induced ROS production and abrogates the phosphorylation of Src, epidermal growth factor receptor and Akt. The combination of bortezomib and saracatinib, a Src inhibitor, synergistically induced hepatoma cell apoptosis. The present study concluded that ROS mediated the activation of the Src-EGFR-Akt cascade by bortezomib. The combination of the Src inhibitor and bortezomib holds promise in the treatment of hepatocellular carcinoma.
\end{abstract}

\section{Introduction}

The proteasome is a cylindrical protein complex that is responsible for the degradation of unnecessary, misfolded or damaged proteins by proteolysis. The basic structure of a proteasome comprises four stacked rings around a central pore (1). Since

Correspondence to: Dr Hui Hua, Laboratory of Stem Cell Biology, West China Hospital, Sichuan University, 1 Ke Yuan 4 Lu, Chengdu, Sichuan 610041, P.R. China

E-mail: hualemon2005@163.com

Key words: bortezomib, Src, Akt, reactive oxygen species the protease active sites of the proteasome are located within the interior surface of its barrel-shaped 20S core, the target protein cannot be degraded until it is recognized by one or two $19 \mathrm{~S}$ regulatory caps. These bind to the outer rings thereby passing the gate formed by the N-termini of its eight $\alpha$ subunits and entering the barrel (2). Ubiquitination is a typical modification that enables a protein substrate to be recognized by the proteasome. Covalent ubiquitination of a lysine residue requires the coordinated reactions of three enzymes, including ubiquitin-activating enzyme, ubiquitin-conjugating enzyme and ubiquitin ligase (3). Although the majority of proteasomal substrates must be polyubiquitinated prior to being degraded, there is ubiquitin-independent degradation of substrates by the proteasome. For example, the proteasomal activation of nuclear factor $\kappa \mathrm{B}(\mathrm{NF}-\kappa \mathrm{B})$ by the processing of p105 into p50 is a ubiquitin-independent process (4). Another ubiquitin-independent proteasome substrate is the enzyme ornithine decarboxylase (5). Certain proteins are subject to either ubiquitin-independent or ubiquitin-dependent degradation, depending on the context. For example, p53 may undergo ubiquitin-independent degradation, although p53 is also subject to ubiquitin-dependent degradation $(6,7)$.

The proteasomal degradation pathway is essential for several cellular processes, including the cell cycle, the regulation of gene expression and responses to cellular stress (8). Therefore, proteasome inhibition is detrimental to cells that are highly dependent on the proteasome degradation system. Notably, studies on PS-341 (also referred to as bortezomib or Velcade ${ }^{\circledR}$ ) demonstrate that levels of systemic proteasome inhibition $\leq 80 \%$ are well tolerated by peripheral blood mononuclear cells and do not cause excessive toxicity in patients $(9,10)$. Bortezomib effectively suppresses certain types of cancer, including multiple myeloma (MM) and mantle cell lymphoma (11). The mechanisms underlying the anti-cancer activities of bortezomib include inhibition of $\mathrm{NF}-\kappa \mathrm{B}$, stabilization of pro-apoptotic proteins, including p53 and Bcl-2-interacting killer, and induction of cellular stress (12). Although bortezomib exhibits antitumor activity in MM, its single agent activity in several types of solid tumor has been modest (13). Thus, it is necessary to identify the mechanisms that determine bortezomib sensitivity and resistance. Previous 
studies have indicated that downregulation of Akt activity is a molecular determinant of bortezomib-induced apoptosis in hepatocellular carcinoma cells (14). Failure in downregulation of cancerous inhibitor of protein phosphatase 2A (CIP2A), an inhibitor of protein phosphatase 2A (PP2A), leads to sustained Akt phosphorylation in bortezomib-treated cancer cells (15). Although inhibition of Akt dephosphorylation may contribute to sustained Akt phosphorylation in bortezomib-treated cancer cells, the upstream signaling elements that induce Akt phosphorylation remain to be elucidated. Phosphatidylinositol-3-OH kinase $(\mathrm{PI} 3 \mathrm{~K})$ is well characterized as a major regulator of Akt activation. Bortezomib may induce Akt phosphorylation through the p38MAPK-Hsp27-MK2 module (16).

The non-receptor tyrosine kinase Src is often associated with malignancy and drug resistance. Src can activate Akt either directly or indirectly to promote its pro-survival signaling functions (17). Src also responds to growth factors and stress, including oxidative stress. While Src may act downstream of epidermal growth factor receptor (EGFR), it also regulates the activity of EGFR (18). In the present study, the role of the Src-EGFR-Akt signaling cascade in bortezomib-induced apoptosis of hepatoma cells was investigated.

\section{Materials and methods}

Reagents. Bortezomib, gefitinib and saracatinib were purchased from LC Laboratories (Woburn, MA, USA). The antioxidant $\mathrm{N}$-acetyl cysteine (NAC) was purchased from Beyotime Institute of Biotechnology (Jiangsu, China). Monoclonal rabbit anti-human anti-EGFR, anti-Akt1 and phosphorylated Akt (S473), anti-Src and phosphorylated Src (Tyr416) antibodies were purchased from Cell Signaling Technology, Inc. (Beverly, MA, USA). Polyclonal rabbit anti-human anti-phosphorylated EGFR (Y1068) antibody was purchased from Epitomics, Inc. (Burlingame, CA, USA). Monoclonal mouse anti-human anti-actin antibody was purchased from Tianjin Sungene Biotechnology Co., Ltd. (Tianjin, China). Horseradish peroxidase goat anti-rabbit immunoglobulin (Ig)G was purchased from Santa Cruz Biothechnology, Inc. (Dallas, TX, USA).

Cell culture. The hepatoma cell lines HepG2, Hep3B and SMMC-7721 were obtained from the Cell Bank, Chinese Academy of Sciences (Shanghai, China). The cells were maintained in Dulbecco's minimal essential medium (Life Technologies, Grand Island, NY, USA) containing 10\% fetal bovine serum (Life Technologies), $100 \mu / \mathrm{ml}$ penicillin and $100 \mu \mathrm{g} / \mathrm{ml}$ streptomycin (North China Pharmaceutical Group Corporation, Shijiazhuang, China). Cells were incubated at $37^{\circ} \mathrm{C}$ in a humidified atmosphere of $5 \% \mathrm{CO}_{2}$.

Western blotting. Cells were lysed with cold radio immunoprecipitation assay lysis buffer containing the protease inhibitors aprotinin and phenylmethanesulfonyl fluoride (Sigma-Aldrich, St. Louis, MO, USA). Cell lysates were collected and protein was obtained by centrifugation at $10,000 \mathrm{x}$ g for $20 \mathrm{~min}$ at $4^{\circ} \mathrm{C}$. Aliquots of proteins were boiled in $4 \mathrm{X}$ loading buffer for $10 \mathrm{~min}$, loaded onto Tris-HCl-polyacrylamide gels and transferred electrophoretically onto a polyvinylidene difluoride membrane (EMD Millipore, Billerica, MA, USA). Membranes were incubated with the above primary antibodies against EGFR, Akt1, phospho-Akt, Src, phospho-Src and phospho-EGFR and horseradish peroxidase-goat anti-rabbit immunoglobulin G secondary antibodies. Membranes were also probed with monoclonal mouse anti-human actin antibody (Tianjin Sungene Biotechnology Co., Ltd.) to normalize the loading of protein among the samples. Detection was performed using chemiluminescent agents (Pierce Biotechnology, Inc., Rockford, IL, USA). Images were captured using an Alpha Innotech FluorChem imaging system (Alpha Innotech, San Leandro, CA, USA).

Detection of ROS. ROS was detected using a Reactive Oxygen Species Assay kit (Beyotime Institute of Biotechnology) and a fluorometric assay using intracellular oxidation of 2',7'-dichlorofluorescein-diacetate (DCFH-DA; Beyotime Institute of Biotechnology), a fluorescence probe, was performed. A total of 5,000 cells/well were plated in a 96-well plate. The cells were treated with or without $100 \mathrm{nM}$ bortezomib in the presence or absence of $5 \mathrm{mM}$ NAC for $24 \mathrm{~h}$, followed by incubation with $10 \mu \mathrm{M}$ DCFH-DA at $37^{\circ} \mathrm{C}$ in $5 \% \mathrm{CO}_{2}$ for $20 \mathrm{~min}$. Following DCFH-DA incubation, the cells were washed three times with serum-free medium to remove any unbound probe. The fluorescence of dichlorofluorescein, which is the oxidized product of DCFH-DA, was measured with excitation and emission wavelengths of 488 and $525 \mathrm{~nm}$, respectively, using a fluorescence detector (Fluoroskan Ascent FL; Thermo Fisher Scientific, Waltham, MA, USA).

Analysis of apoptotic cells. Cell apoptosis was detected using a terminal deoxynucleotidyl transferase dUTP nick end labeling (TUNEL) assay (Beyotime Institute of Biotechnology). Cells were plated onto tissue culture plates, treated with or without $100 \mathrm{nM}$ bortezomib and in the presence or absence of $5 \mu \mathrm{M}$ saracatinib for $48 \mathrm{~h}$. The cells were incubated with TUNEL solution and 4',6-diamidino-2-phenylindole (DAPI) solution at $37^{\circ} \mathrm{C}$ for $60 \mathrm{~min}$ without light. Apoptotic cells were then examined under a fluorescence microscope (DMI4000B; Leica Microsystems GmbH, Wetzlar, Germany). Green fluorescence was observed in the nuclei of apoptotic cells. All cells demonstrated blue fluorescence for DAPI. Quantification of all cells and apoptotic cells in the same fields was performed by obtaining images in random fields and counting at least 300 cells in three random fields in each well.

\section{Results}

Bortezomib induces EGFR, Src and Akt phosphorylation. To detect the effects of bortezomib on the phosphorylation of Akt and its associated kinases, including EGFR and Src in hepatoma cells, SMMC-7721 cells were treated with or without bortezomib, followed by western blot analysis of EGFR, Src and Akt phosphorylation. Bortezomib induced the phosphorylation of EGFR, Src and Akt in a dose-dependent manner, while the total levels of EGFR, Src and Akt were unaffected by bortezomib (Fig. 1). Similar results were detected in the Hep3B and HepG2 cells (Fig. 1).

Gefitinib decreases bortezomib-induced phosphorylation of Src and Akt. To determine the effect of EGFR phosphorylation on the bortezomib-induced phosphorylation of Src 

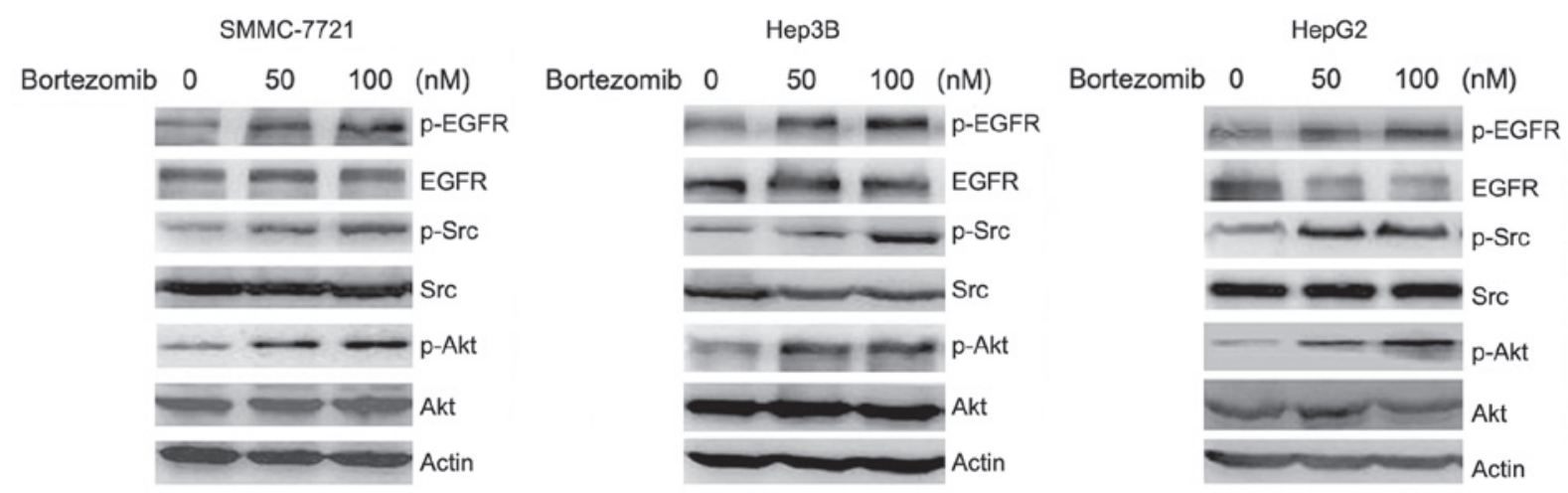

Figure 1. Bortezomib induces EGFR, Src and Akt phosphorylation in hepatoma cells. SMMC-7721, Hep3B and HepG2 cells were treated with bortezomib at the indicated dose for $24 \mathrm{~h}$, followed by western blot analysis of EGFR, Src and Akt phosphorylation. EGFR, epidermal growth factor receptor; p-, phosphorylated.
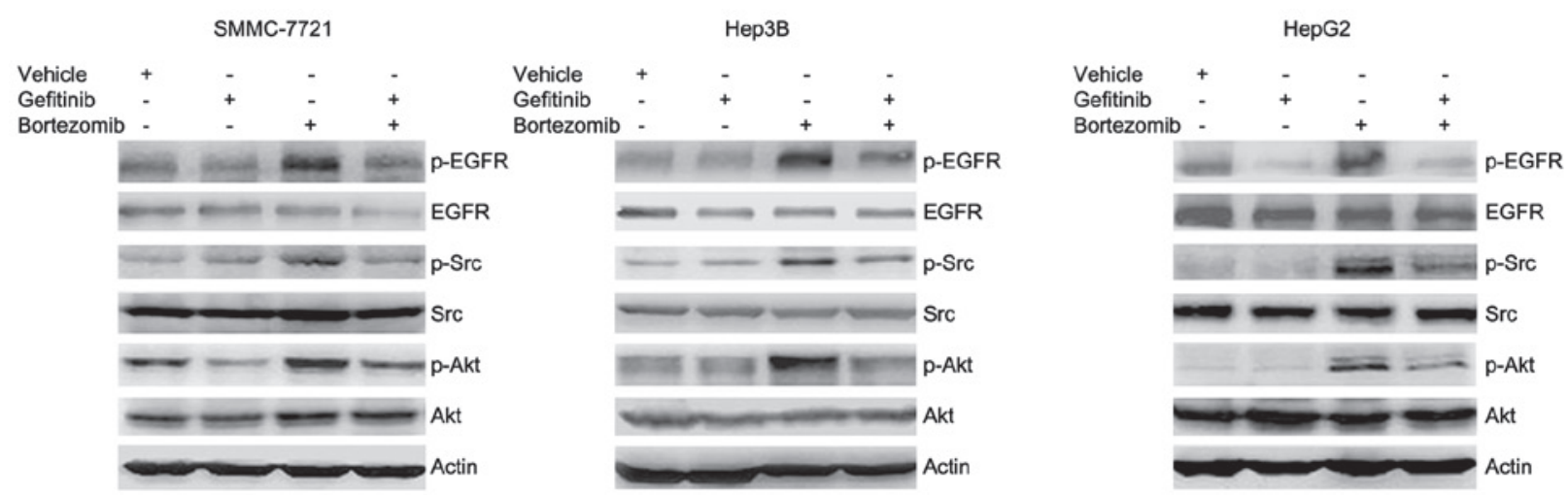

Figure 2. Gefitinib inhibits bortezomib-induced EGFR, Src and Akt phosphorylation. SMMC-7721, Hep3B and HepG2 cells were treated with or without $10 \mu \mathrm{M}$ gefitinib and $100 \mathrm{nM}$ bortezomib for $24 \mathrm{~h}$, followed by western blot analysis of EGFR, Src and Akt phosphorylation. EGFR, epidermal growth factor receptor; p-, phosphorylated.
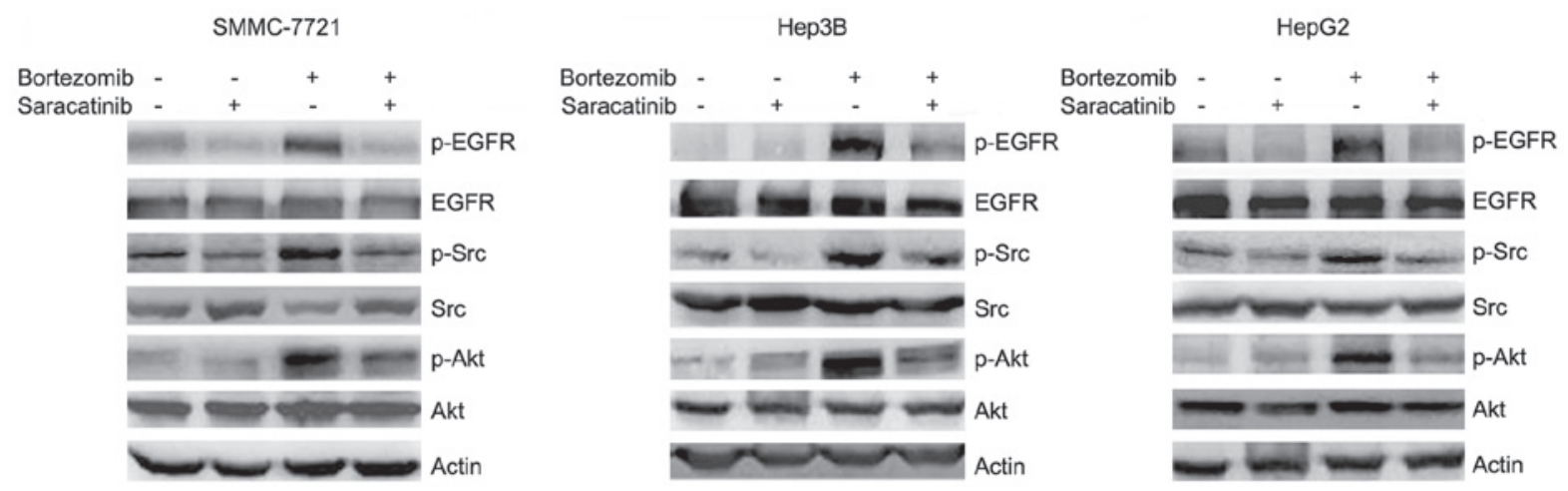

Figure 3. Saracatinib decreases bortezomib-induced EGFR, Src and Akt phosphorylation. SMMC-7721, Hep3B and HepG2 cells were treated with or without $1.5 \mu \mathrm{M}$ saracatinib and $100 \mathrm{nM}$ bortezomib for $24 \mathrm{~h}$, followed by western blot analysis of EGFR, Src and Akt phosphorylation. EGFR, epidermal growth factor receptor; p-, phosphorylated.

and Akt, the effects of gefitinib, an EGFR inhibitor, on the bortezomib-induced phosphorylation of Src and Akt were examined. Treatment of SMMC-7721 cells with gefitinib eliminated bortezomib-induced phosphorylation of EGFR, Src and Akt (Fig. 2). Similar results were detected in the Hep3B and HepG2 cells (Fig. 2). These results indicated that the activation of EGFR by bortezomib may have contributed to the phosphorylation of Src and Akt.
Src inhibitor abrogates bortezomib-induced phosphorylation of EGFR and Akt. Although activation of EGFR may lead to phosphorylation of Src, Src also mediates the phosphorylation of EGFR and modulates receptor function. To determine the effect of Src on the induction of EGFR and Akt phosphorylation by bortezomib, the effects of saracatinib, a Src inhibitor, on the bortezomib-induced phosphorylation of EGFR and Akt were determined. Treatment of SMMC-7721 
A

SMMC-7721
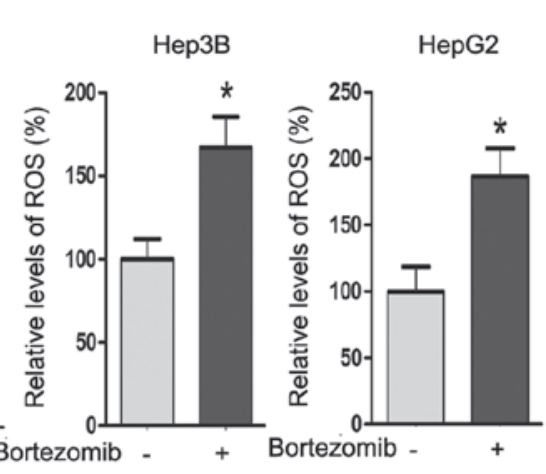

B

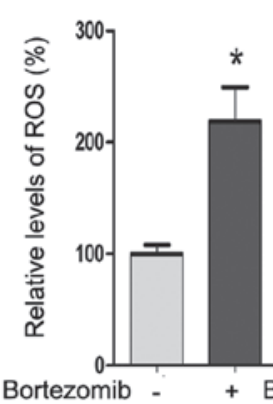

C

SMMC-7721
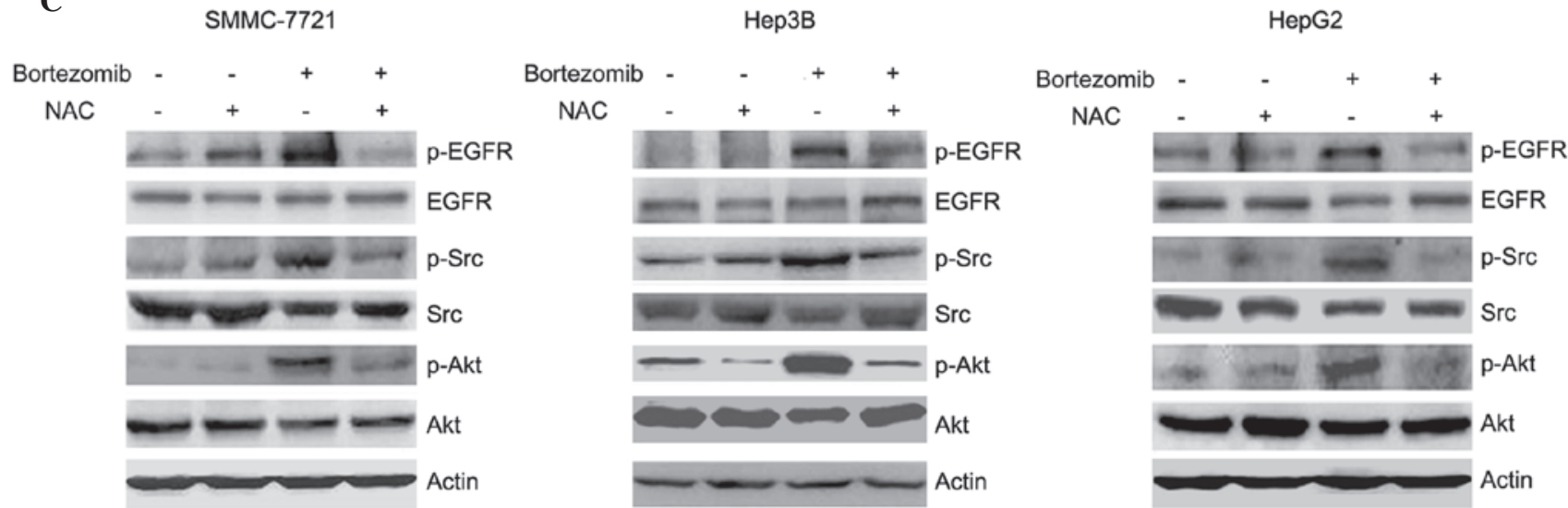

Figure 4. ROS mediate the induction of Src, EGFR and Akt phosphorylation by bortezomib. (A) SMMC-7721, Hep3B and HepG2 cells treated with or without $100 \mathrm{nM}$ bortezomib for $24 \mathrm{~h}$, followed by detection of intracellular ROS. The relative levels of ROS were plotted. Values are expressed as the mean \pm SD "P<0.05 vs. control. (B) SMMC-7721 and Hep3B cells treated with or without $5 \mathrm{mM} \mathrm{NAC}$ and $100 \mathrm{nM}$ bortezomib for $24 \mathrm{~h}$, followed by detection of intracellular ROS. The relative levels of ROS were plotted. Values are expressed as the mean \pm SD. ${ }^{*}<<0.05$. (C) SMMC-7721, Hep3B and HepG2 cells treated with or without $5 \mathrm{mM} \mathrm{NAC}$ and $100 \mathrm{nM}$ bortezomib for $24 \mathrm{~h}$, followed by western blot analysis of EGFR, Src and Akt phosphorylation. ROS, reactive oxygen species; EGFR, epidermal growth factor receptor; p-, phosphorylated; SD, standard deviation; NAC, N-acetyl cysteine.

cells with saracatinib decreased the bortezomib-induced phosphorylation of EGFR and Akt (Fig. 3). Similar results were observed in the Hep3B and HepG2 cells (Fig. 3). These results indicated that phosphorylation of Src also contributed to the bortezomib-induced phosphorylation of EGFR and Akt.

ROS mediates bortezomib-induced phosphorylation of EGFR, Src and Akt. Bortezomib reportedly causes the accumulation of ROS in cancer cells. Treatment of hepatoma cells with bortezomib led to an increase in the levels of ROS (Fig. 4A). NAC, an antioxidant agent, inhibited the induction of ROS by bortezomib (Fig. 4B). To determine whether ROS may trigger phosphorylation of EGFR, Src and Akt, SMMC-7721 cells were treated with or without bortezomib and NAC, followed by western blot analysis of EGFR, Src and Akt phosphorylation. Treatment with NAC abrogated the induction of EGFR, Src and Akt phosphorylation (Fig. 4C), suggesting that ROS mediated the effects of bortezomib on the phosphorylation of EGFR, Src and Akt. Similar results were detected in Hep3B and HepG2 cells (Figure 4C).

As ROS mediated the effects of bortezomib on the phosphorylation of EGFR, Src and Akt (Fig. 4), the ability of the ROS scavenger NAC to sensitize hepatoma cells to bortezomib was examined. A combination of bortezomib and NAC did not increase the sensitivity of hepatoma cells to bortezomib (data not shown).

Inhibition of Src sensitizes hepatoma cells to bortezomib. The effect of saracatinib on cell proliferation and apoptosis in bortezomib-treated hepatoma cells was then determined. Cellular proliferation was detected by EdU labeling. No significant difference was identified in the rate of cell proliferation between bortezomib-treated and bortezomib and sarcatinib-treated cells (data not shown). However, treatment of SMMC-7721 cells with saracatinib and bortezomib synergistically induced apoptosis, while induction of apoptosis was poor when either were used alone (Fig. 5A). Similar results were observed in the Hep3B and HepG2 cells (Fig. 5B and C). These results demonstrated that inhibition of Src may have sensitized hepatoma cells to bortezomib.

\section{Discussion}

The proteasome inhibitor bortezomib is an anticancer agent that has been administered in the clinic. Given the importance of proteasomes in multiple cellular processes, proteasome inhibitors hold promise in treating cancer. It is well established that bortezomib exerts its antitumor activity against cancer cells through the inhibition of NF- $\mathrm{kB}$ activation by preventing I $\kappa$ B degradation (19). Although bortezomib 
A
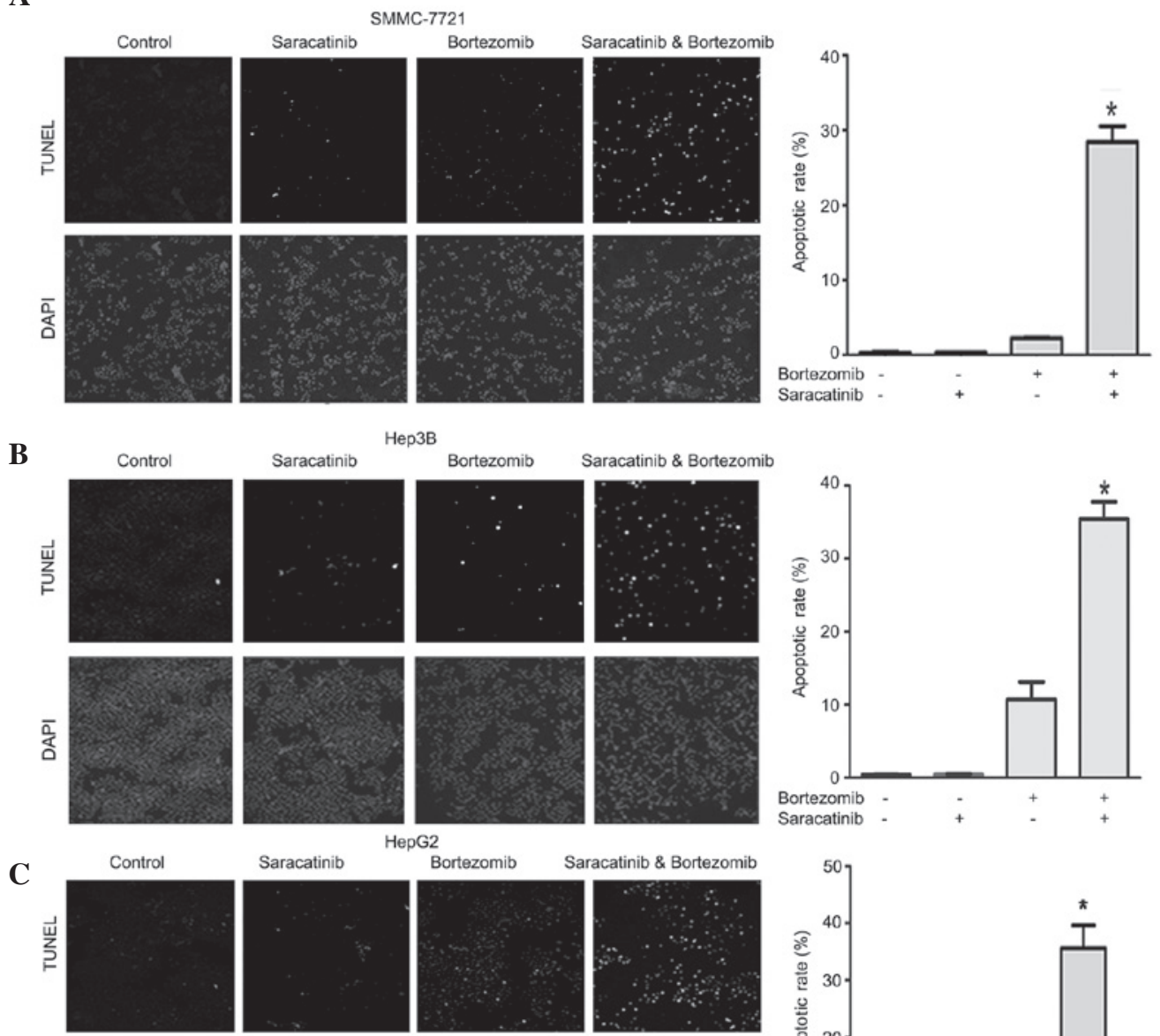

Saracatinib
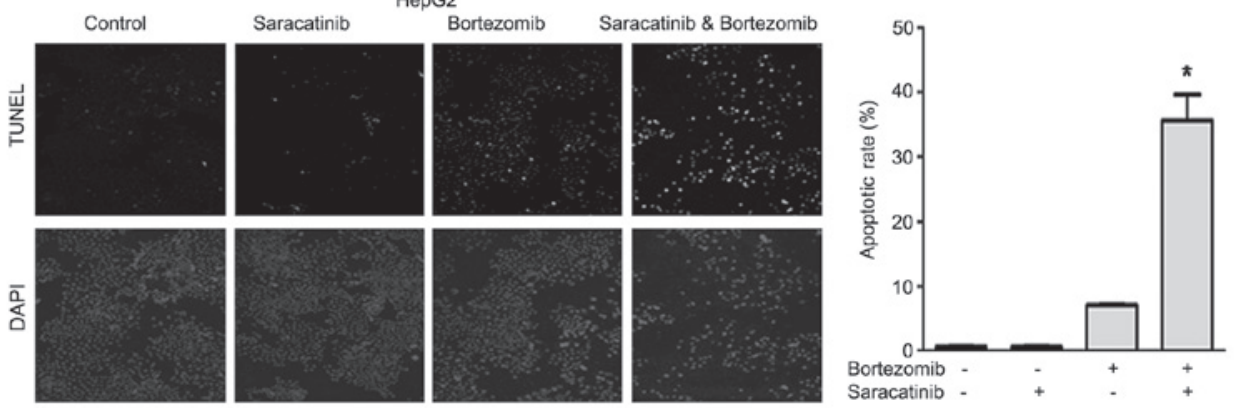

Figure 5. Saracatinib and bortezomib synergistically induce hepatoma cell apoptosis. (A) SMMC-7721 cells treated with or without $5 \mu \mathrm{M}$ saracatinib and $100 \mathrm{nM}$ bortezomib for $48 \mathrm{~h}$, followed by detection of apoptosis by TUNEL. The apoptotic rate was plotted and values are expressed as the mean \pm SD. ${ }^{*} \mathrm{P}<0.05$ vs. control. (B) Hep3B cells treated with or without $5 \mu \mathrm{M}$ saracatinib and $100 \mathrm{nM}$ bortezomib for $48 \mathrm{~h}$, followed by detection of apoptosis by TUNEL. The apoptotic rate was plotted and the values are expressed as the mean $\pm \mathrm{SD}$. ${ }^{*} \mathrm{P}<0.05$. (C) HepG2 cells treated with or without $5 \mu \mathrm{M}$ saracatinib and $100 \mathrm{nM}$ bortezomib for $48 \mathrm{~h}$, followed by detection of apoptosis by TUNEL. The apoptotic rate was plotted. Values are expressed as the mean \pm SD. ${ }^{*} \mathrm{P}<0.05$. DAPI, 4',6-diamidino-2-phenylindole; TUNEL, terminal deoxynucleotidyl transferase dUTP nick end labeling; SD, standard deviation.

inhibits solid tumors in pre-clincial studies, its clinical activity against solid tumors is limited (20). Numerous cancer cells have little sensitivity to bortezomib or become resistant following initial responsiveness to this agent. The phosphorylation of Akt is one of the mechanisms underlying bortezomib resistance. Activation of the PI3K/Akt pathway is important in basic cellular functions, including survival, proliferation, migration and differentiation, that underlie the biology of human cancer (21). The activation of Akt may be associated with the resistance of cellular stress, as a mechanism for cells to avoid death. Akt regulates the survival and apoptosis of cells through activation of downstream proteins, including the mammalian target of rapamycin (22). CIP2A is an oncoprotein that promotes cell growth and tumor formation through the stabilization of c-Myc (23). Previous studies have demonstrated that CIP2A is important in bortezomib-induced apoptosis $(15,24)$. Bortezomib increases the activity of PP2A and downregulates CIP2A in a dose and time-dependent manner in sensitive hepatocellular carcinoma (HCC) cells (24). In addition, overexpression of CIP2A upregulates phospho-Akt and protects cells from bortezomib-induced apoptosis (24).

Bortezomib increases the activity of EGFR in squamous cell, pancreatic and renal carcinoma as well as in head and neck cancer cells, whereas the activity of EGFR is inhibited by bortezomib in breast cancer cells (25-27). The increase in EGFR activity appears to contribute to the phosphorylation of Akt in bortezomib-treated cancer cells (28). The synergistic anti-proliferative and pro-apoptotic activity of combined therapy with bortezomib and anti-EGFR drugs is observed in human cancer cells $(25,29)$. Sorafenib is a small molecule initially developed as an inhibitor of Raf-1. Subsequently, it 
was demonstrated to inhibit the activities of B-Raf, vascular endothelial growth factor receptor and platelet-derived growth factor receptor (30). The combination of sorafenib and bortezomib demonstrates synergy in HCC through PP2A-dependent inactivation of Akt (31). Saracatinib is an oral, tyrosine kinase inhibitor selective for Src. Preliminary data demonstrates that saracatinib has clinical benefits for patients with advanced disease (32). The present study demonstrated that bortezomib induced the phosphorylation of Src. While Src is an effector downstream of EGFR, it also acts upstream of EGFR (33), thus, Src and EGFR form a regulatory circuit. The present study demonstrated that inhibition of EGFR led to a decrease in bortezomib-induced phosphorylation of Src and inhibition of Src decreased bortezomib-induced phosphorylation of EGFR. Inhibitors of EGFR and Src abrogated the bortezomib-induced phosphorylation of Akt, suggesting that EGFR and Src can be ascribed to the bortezomib-induced phosphorylation of Akt. Although it is known that EGFR contributes to bortezomib-induced phosphorylation of Akt, how EGFR is activated by bortezomib remains to be elucidated. Previous studies have indicated that bortezomib may induce the accumulation of ROS (34) and it is known that ROS may induce the phosphorylation of Src (35). The present study demonstrated that ROS mediated the induction of Src phosphorylation by bortezomib. Thus, ROS may be a key mediator of bortezomib-induced phosphorylation of Src, EGFR and Akt. Although ROS triggers these protective effects, an ROS scavenger does not affect bortezomib-induced cell death (data not shown). One possibility is that ROS itself contributed to bortezomib-induced cell death, however, it also triggered a protective response. Thus, the effect of ROS scavengers on bortezomib-induced cell death may be neutral. The effect of bortezomib on cancer cell death can be enhanced by inhibitors of EGFR and Akt $(2,36,37)$. The present study demonstrated that a Src inhibitor also enhanced bortezomib-induced hepatoma cell apoptosis. Therefore, the combination of bortezomib with a Src inhibitor holds promise in treating hepatoma.

\section{Acknowledgements}

The authors would like to thank the National Natural Science Foundation of China (grant no. 81272215) for their support.

\section{References}

1. Walz J, Erdmann A, Kania M, Typke D, Koster AJ and Baumeister W: 26S proteasome structure revealed by three-dimensional electron microscopy. J Struct Biol 121: 19-29, 1998.

2. Kim HM, Yu Y and Cheng Y: Structure characterization of the 26S proteasome. Biochim Biophys Acta 1809: 67-79, 2011.

3. Pickart CM and Eddins MJ: Ubiquitin: structures, functions, mechanisms. Biochim Biophys Acta 1695: 55-72, 2004.

4. Pereira SG and Oakley F: Nuclear factor- $\kappa$ B1: regulation and function. Int J Biochem Cell Bio 40: 1425-1430, 2008.

5. Zhang M,Pickart CM and Coffino P: Determinants of proteasome recognition of ornithine decarboxylase, a ubiquitin-independent substrate. EMBO J 22: 1488-1496, 2003.

6. Tsvetkov P, Reuven N and Shaul Y: Ubiquitin-independent p53 proteasomal degradation. Cell Death Differ 17: 103-108, 2010

7. Lee YS, Wan J, Kim BJ, Bae MA and Song BJ Ubiquitin-dependent degradation of p53 protein despite phosphorylation at its $\mathrm{N}$ terminus by acetaminophen. J Pharmacol Exp Ther 317: 202-208, 2006.
8. Jariel-Encontre I, Bossis $G$ and Piechaczyk M: Ubiquitin-independent degradation of proteins by the proteasome. Biochim Biophys Acta 1786: 153-177, 2008.

9. Papandreou $\mathrm{CN}$ and Logothetis CJ: Bortezomib as a potential treatment for prostate cancer. Cancer Res 64: 5036-5043, 2004.

10. Richardson PG and Anderson KC: Bortezomib: a novel therapy approved for multiple myeloma. Clin Adv Hematol Oncol 1: 596-600, 2003.

11. O'Connor OA: Marked clinical activity of the proteasome inhibitor bortezomib in patients with follicular and mantle-cell lymphoma. Clin Lymphoma Myeloma 6: 191-199, 2005.

12. Ko BS, Chang TC, Chen $\mathrm{CH}$, et al: Bortezomib suppresses focal adhesion kinase expression via interrupting nuclear factor-kappa B. Life Sci 86: 199-206, 2010.

13. Lara PN, Jr, Davies AM, Mack PC, et al: Proteasome inhibition with PS-341 (bortezomib) in lung cancer therapy. Semin Oncol 31: 40-46, 2004.

14. Chen KF, Yeh PY, Yeh KH, Lu YS, Huang SY and Cheng AL: Downregulation of phospho-Akt is a major molecular determinant of bortezomib-induced apoptosis in hepatocellular carcinoma cells. Cancer Res 68: 6698-6707, 2008.

15. Lin YC, Chen KC, Chen CC, Cheng AL and Chen KF: CIP2A-mediated Akt activation plays a role in bortezomib-induced apoptosis in head and neck squamous cell carcinoma cells. Oral Oncol 48: 585-593, 2012.

16. McCubrey JA, Steelman LS, Abrams SL, et al: Roles of the RAF/MEK/ERK and PI3K/PTEN/AKT pathways in malignant transformation and drug resistance. Adv Enzyme Regul 46: 249-279, 2006

17. Haynes MP, Li L, Sinha D, et al: Src kinase mediates phosphatidylinositol 3-kinase/Akt-dependent rapid endothelial nitric-oxide synthase activation by estrogen. J Biol Chem 278: 2118-2123, 2003

18. Mao W, Irby R, Coppola D, et al: Activation of c-Src by receptor tyrosine kinases in human colon cancer cells with high metastatic potential. Oncogene 15: 3083-3090, 1997.

19. Yang F, Jove V, Chang S, et al: Bortezomib induces apoptosis and growth suppression in human medulloblastoma cells, associated with inhibition of AKT and NF- $\mathrm{BB}$ signaling and synergizes with an ERK inhibitor. Cancer Biol Ther 13: 349-357, 2012.

20. Markovic SN, Geyer SM, Dawkins F, et al: A phase II study of bortezomib in the treatment of metastatic malignant melanoma. Cancer 103: 2584-2589, 2005.

21. Vivanco I and Sawyers CL: The phosphatidylinositol 3-kinase AKT pathway in human cancer. Nat Rev Cancer 2: 489-501, 2002.

22. Hay N: The Akt-mTOR tango and its relevance to cancer. Cancer Cell 8: 179-183, 2005.

23. Junttila MR, Puustinen P, Niemelä M, et al: CIP2A inhibits PP2A in human malignancies. Cell 130: 51-62, 2007.

24. Chen K, Liu C, Lin Y, et al: CIP2A mediates effects of bortezomib on phospho-Akt and apoptosis in hepatocellular carcinoma cells. Oncogene 29: 6257-6266, 2010.

25. An J and Rettig MB: Epidermal growth factor receptor inhibition sensitizes renal cell carcinoma cells to the cytotoxic effects of bortezomib. Mol Cancer Ther 6: 61-69, 2007.

26. Lorch JH, Thomas TO and Schmoll HJ: Bortezomib inhibits cell-cell adhesion and cell migration and enhances epidermal growth factor receptor inhibitor-induced cell death in squamous cell cancer. Cancer Res 67: 727-734, 2007.

27. Codony-Servat J, Tapia MA, Bosch M, et al: Differential cellular and molecular effects of bortezomib, a proteasome inhibitor, in human breast cancer cells. Mol Cancer Ther 5: 665-675, 2006.

28. Sloss CM, Wang F, Liu R, et al: Proteasome inhibition activates epidermal growth factor receptor (EGFR) and EGFR-independent mitogenic kinase signaling pathways in pancreatic cancer cells. Clin Cancer Res 14: 5116-5123, 2008.

29. Cascone T, Morelli MP, Morgillo F, et al: Synergistic anti-proliferative and pro-apoptotic activity of combined therapy with bortezomib, a proteasome inhibitor, with anti-epidermal growth factor receptor (EGFR) drugs in human cancer cells. J Cell Physiol 216: 698-707, 2008.

30. Wilhelm SM, Adnane L, Newell P, Villanueva A, Llovet JM and Lynch M: Preclinical overview of sorafenib, a multikinase inhibitor that targets both Raf and VEGF and PDGF receptor tyrosine kinase signaling. Mol Cancer Ther 7: 3129-3140, 2008.

31. Chen KF, Yu HC, Liu TH, Lee SS, Chen PJ and Cheng AL: Synergistic interactions between sorafenib and bortezomib in hepatocellular carcinoma involve PP2A-dependent Akt inactivation. J Hepatol 52: 88-95, 2010. 
32. Saad F and Lipton A: SRC kinase inhibition: targeting bone metastases and tumor growth in prostate and breast cancer. Cancer Treat Rev 36: 177-184, 2010.

33. Martinez Flores K, Uribe Marin BC, Souza Arroyo V, et al: Hepatocytes display a compensatory survival response against cadmium toxicity by a mechanism mediated by EGFR and Src. Toxicol In Vitro 27: 1031-1042, 2013.

34. Yu C, Rahmani M, Dent P and Grant S: The hierarchical relationship between MAPK signaling and ROS generation in human leukemia cells undergoing apoptosis in response to the proteasome inhibitor Bortezomib. Exp Cell Res 295: 555-566, 2004
35. Vasant C, Rajaram R and Ramasami T: Apoptosis of lymphocytes induced by chromium (VI/V) is through ROS-mediated activation of Src-family kinases and caspase-3. Free Radic Biol 35: 1082-1100, 2003.

36. Morgillo F, D'Aiuto E, Troiani T, et al: Antitumor activity of bortezomib in human cancer cells with acquired resistance to anti-epidermal growth factor receptor tyrosine kinase inhibitors. Lung Cancer 71: 283-290, 2011.

37. Kim A, Park S, Lee JE, et al: The dual PI3K and mTOR inhibitor NVP-BEZ235 exhibits anti-proliferative activity and overcomes bortezomib resistance in mantle cell lymphoma cells. Leuk Res 36: 912-920, 2012. 